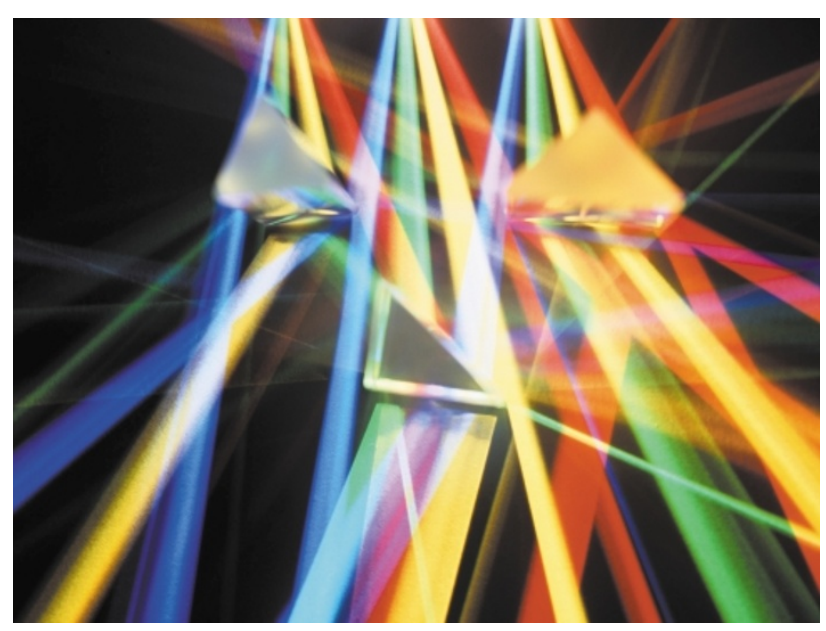

DEVELOPMENTAL BIOLOGY

\section{A converging pattern}

Converting a morphogenetic field into a defined tissue or organ involves the precise interaction of a complex network of genes. The defining event in such a process is the discrete expression of socalled 'field-selector genes', which, in the case of the fruitfly wing, ensure that the cluster of cells in which they are expressed develop into a wing rather than into another organ. But how do selector genes orchestrate the development of complex structures and how do they impart specificity to globally deployed signalling pathways such as Decapentaplegic (Dpp) and Notch, which pattern the wing as well as many other organs? Investigating how selector genes direct the expression of field-specific responses has been the focus of a recent study by Guss et al. Their results show that the expression of wing-specific responses requires both wing-specific selector genes and commonly deployed signalling pathways.

The selector gene complex that specifies wing identity consists of two proteins, Vestigial $(\mathrm{Vg})$ and Scalloped (Sd). The sd gene encodes a DNA-binding protein that is required cellautonomously for the expression of wing-patterning genes such as cut, spalt and $v g$. Sd binds directly to the cis-regions of the target genes it regulates. In addition, mutating the Sd-binding sites in the promoters of $c u t$, spalt and $v g$ eliminates their expression. It is therefore possible that, in the wing, field-selector genes control directly the expression of all target genes, rather than controlling only a few members of the gene-regulatory network. However, $c u t$, spalt and $v g$ are also known targets of the Notch and Dpp signalling pathways. This observation led Guss et al. to test whether field-specific genes and commonly deployed signalling pathways pattern the wing field by converging on the control regions of the same genes. Indeed, only when binding sites for both selector and signalling genes are present on a synthetic promoter construct can wing-specific gene expression and patterning occur.

This study has made a step towards clarifying the relationship between two genetic systems that are required to specify and pattern a structure, by showing that both systems depend on each other. Although these results are specific to wing development, an obligatory combinatorial input on target promoters might be a general mechanism by which selector genes work with signalling pathways to control the extensive genetic network that specifies the development of complex structures.

Tanita Casci

6) References and links

ORIGINAL RESEARCH PAPER Guss, K. A. et al. Control of a genetic regulatory network by a selector gene. Science 12 April 2001 (10.1126/science.1058312)

FURTHER READING Mann, R. S. \& Morata, G. The developmental and molecular biology of genes that subdivide the body of Drosophila. Annu. Rev. Cell Dev. Biol. 16, 243-271 (2000) WEB SITE Sean Carroll's lab

H I G H L I G H T S

MOUSE MODELS

\section{A hit-and-run on sporadic cancer}

Mouse models for tumorigenesis have traditionally mimicked hereditary rather than sporadic cancers, as it is relatively straightforward to breed mice with a germline mutation that predisposes them to cancer. However, the development of a modified 'hit-andrun' gene-targeting technique, described by Jacks and colleagues in Nature, is set to change this.

The technique targets the K-ras oncogene - which is mutated in $30 \%$ of human tumours - and the advance comes not in the 'hit' step, in which the mutated allele is inserted into the genome of embryonic stem cells, but in the 'run' step. The authors capitalized on the fact that mitotic recombination occurs $10^{-3}-10^{-7}$ times per cell division. By building a construct that could recombine to produce an oncogenic ras gene, they can trigger tumorigenesis spontaneously in vivo — as would occur in sporadic cancer.

The authors generated two constructs, $K-r a s^{L A 1}$ and $K-r a s^{L A 2}$. Each carried two copies of exon 1 , in which codon 12 of one or both of the copies, respectively, was mutated from glycine to aspartic acid, leading to a constitutively active K-ras protein. Recombination in cis resulted in oncogenic $\mathrm{K}$-ras in $50 \%$ of recombination events in $\mathrm{K}$-ras ${ }^{L A l}$ mice, but in $100 \%$ of events in $K$-ras ${ }^{L A 2}$ mice. $K$-ras ${ }^{L A}$ mice had reduced lifespans $-K$-ras ${ }^{L A 2}$ mice the shortest - compared with wildtype mice, and developed many tumours. Both strains had a similar tumour spectrum, and all mutants developed tumours that histopathologically resembled human non-smallcell lung cancer. Approximately $30 \%$ of the mice also developed thymic lymphoma and skin papilloma. Biochemical analyses showed that tumour cells carried the recombined, oncogenic ras allele.

Surprisingly, the mice did not develop carcinomas of the colon or pancreas, cancers frequently associated with the $K$-ras oncogene in humans. As carcinomas with $K$-ras mutations also often have defects in the p53 pathway, the authors crossed $K-r a s^{L A l}$ mice with Trp53-deleted mice. Trp $53^{+/}$and $\operatorname{Tr} p 53^{--}$mice, when carrying the $K$ $\mathrm{ras}^{L A 1}$ allele, showed a further reduction in survival compared with parental strains. Although these mice had a broader range of tumours - $30 \%$ developed haemangiosarcomas and fibrosarcomas - colonic and pancreatic tumours still did not occur. Goals for the future will therefore be to understand why this oncogene so consistently results in lung cancer in mice and to refine the technique to create models of the tumour types that occur in humans with $\mathrm{K}$-ras mutations.

With new initiatives to develop better mouse models of cancer, this is an exciting time for the cancer research community. This study by Johnson et al. is an early fruit of such an initiative, and no doubt more will follow in the future.

Emma Greenwood, Associate Editor, Nature Reviews Cancer

(D) References and links ORIGINAL RESEARCH PAPER Johnson, L. et al. Somatic activation of the $K$-ras oncogene causes early onset lung cancer in mice. Nature $\mathbf{4 1 0}$, 1111-1116 (2001) WEB SITE Mouse Models of Human Cancer Consortium

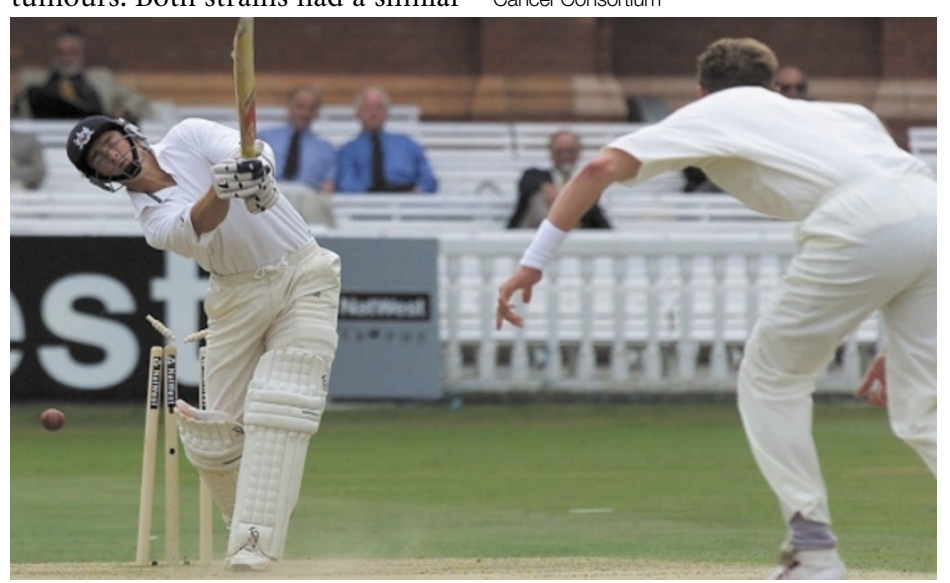

